\title{
Imaging Findings Associated with Cognitive Performance in Primary Lateral Sclerosis and Amyotrophic Lateral Sclerosis
}

\author{
Avner Meoded $^{a} \quad$ Justin Y. Kwan a, ${ }^{a}$ Tracy L. Peters ${ }^{a, i} \quad$ Edward D. Huey ${ }^{a, c, d}$ \\ Laura E. Danielian $^{a}$ Edythe Wiggs ${ }^{a}$ Arthur Morrissette ${ }^{a}$ Tianxia Wu ${ }^{a}$ \\ James W. Russell ${ }^{\mathrm{b}} \quad$ Elham Bayat ${ }^{\mathrm{e}} \quad$ Jordan Grafman ${ }^{\mathrm{a}}$ f-h Mary Kay Floeter $^{\mathrm{a}}$ \\ a National Institute of Neurological Disorders and Stroke, National Institutes of Health, Bethesda, \\ Md., b Department of Neurology, Maryland ALS Clinic, University of Maryland, Baltimore, Md., \\ Departments of ${ }^{\mathrm{C}}$ Psychiatry and ${ }^{\mathrm{d}}$ Neurology, Columbia University, New York, N.Y., ${ }^{\text {e Department of }}$ \\ Neurology, ALS Clinic, George Washington University, Washington, D.C., and ${ }^{\mathrm{f}}$ Department of Physical \\ Medicine and Rehabilitation, ${ }^{9}$ Department of Psychiatry and Behavioral Sciences and Cognitive \\ Neurology, Northwestern University Medical School, and h ${ }^{\mathrm{h}}$ Department of Psychology, Northwestern

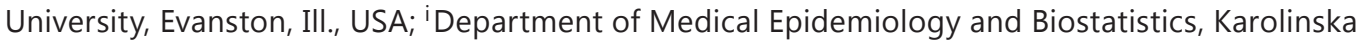 \\ Institute, Stockholm, Sweden
}

\section{Key Words}

Motor neuron disease $\cdot$ Executive function - Diffusion tensor imaging

\begin{abstract}
Introduction: Executive dysfunction occurs in many patients with amyotrophic lateral sclerosis (ALS), but it has not been well studied in primary lateral sclerosis (PLS). The aims of this study were to (1) compare cognitive function in PLS to that in ALS patients, (2) explore the relationship between performance on specific cognitive tests and diffusion tensor imaging (DTI) metrics of white matter tracts and gray matter volumes, and (3) compare DTI metrics in patients with and without cognitive and behavioral changes. Methods: The Delis-Kaplan Executive Function System (D-KEFS), the Mattis Dementia Rating Scale (DRS-2), and other behavior and mood scales were administered to 25 ALS patients and 25 PLS patients. Seventeen of the PLS patients, 13 of the ALS patients, and 17 healthy controls underwent structural magnetic resonance imaging (MRI) and DTI. Atlas-based analysis using MRI Studio software was used to measure fractional anisotropy, and axial and radial diffusivity of selected white matter tracts. Voxel-based morphometry was used to assess gray matter volumes. The relationship between diffusion properties of selected association and commissural white matter and performance on executive function and memory tests was explored using a linear regression model. Results: More ALS than PLS patients had abnormal scores on the DRS-2. DRS-2 and
\end{abstract}


Meoded et al.: Imaging Findings Associated with Cognitive Performance in Primary Lateral Sclerosis and Amyotrophic Lateral Sclerosis

D-KEFS scores were related to DTI metrics in several long association tracts and the callosum. Reduced gray matter volumes in motor and perirolandic areas were not associated with cognitive scores. Conclusion: The changes in diffusion metrics of white matter long association tracts suggest that the loss of integrity of the networks connecting fronto-temporal areas to parietal and occipital areas contributes to cognitive impairment.

(c) 2013 S. Karger AG, Base

\section{Introduction}

Many patients with amyotrophic lateral sclerosis (ALS) develop impairment in executive functions such as reasoning, organization, attention, judgment, and mental flexibility [1-3]. These cognitive changes resemble those in fronto-temporal lobar dementia (FTLD), but may not be severe enough to fulfill the clinical criteria of Neary et al. [4] for FTLD; however, more recently proposed diagnostic criteria may include more patients [5]. In ALS, consensus criteria have been proposed for a milder degree of cognitive impairment (ALSci). Patients are classified as ALSci when they perform at the 5th percentile or below compared to age- and education-matched norms on at least two standardized tests of executive functioning [6]. Similar criteria were developed for behavioral impairment in ALS patients (ALSbi) who have at least two diagnostic features from the FTLD criteria, such as loss of inhibition, impaired insight, apathy, or impaired regulation of social conduct [6]. Primary lateral sclerosis (PLS) is a rare form of motor neuron disorder [7-9]; however, cognitive functioning in PLS has been less well studied than in ALS. Two retrospective studies $[10,11]$ reported a variety of cognitive deficits in some PLS patients, and one prospective study found similar proportions of PLS and ALS patients who had deficits in verbal fluency and memory [12].

In FTLD, different clinical syndromes have been associated with differing patterns of gray matter loss in regions of the brain [13-15]. Frontal lobe atrophy has been reported in ALS patients with fronto-temporal dementia [16] and in ALS patients with lower scores on the revised ALS Functional Rating Scale (ALSFRS-R) [17], but it is unclear whether patients with ALSci or ALSbi, both of which are milder forms of impairment, exhibit changes in similar brain regions as patients with more severe cognitive deficits. One study of ALS patients with verbal fluency defects found reduced volume of frontal white matter [18], and another reported associations between performance on particular neuropsychological tests and a variety of diffusion properties of white matter association tracts in a cohort of 16 ALS patients that included 2 ALSci patients [19]. Another study found left prefrontal and parietal cortical thinning in ALS patients with poorer performance on a sorting task [20]. Associations between imaging and cognitive findings have not been similarly investigated in PLS patients.

The aims of our study were (1) to determine whether cognitive and behavioral functions are impaired in PLS patients to a similar extent as in ALS patients, (2) to explore the relationship between deficits in performance on specific cognitive tests and diffusion tensor imaging (DTI) metrics of white matter association tracts and gray matter volumetric changes, and (3) to compare DTI metrics in patients with and without cognitive and behavioral changes.

\section{Methods}

Subjects

Twenty-five patients who fulfilled the criteria of Pringle et al. [7] for PLS, 25 patients with probable or definite ALS by the revised El Escorial criteria [21], and 17 age-matched healthy controls participated in the study, which was approved by the institutional review board. All 
subjects gave written informed consent for the protocol. In accordance with the protocol, PLS and ALS patients also designated a caretaker as a surrogate decision maker who provided neurobehavioral information. Only ALS and PLS patients underwent the neuropsychological testing battery.

\section{Clinical Evaluation}

All ALS and PLS patients and healthy controls had a detailed history and neurological examination by one of the neurologist coauthors. All patients underwent diagnostic testing, such as EMG, to confirm that they fulfilled the El Escorial criteria for probable or definite ALS [21] or the criteria for PLS from Pringle et al. [7]. The ALSFRS-R was recorded for all subjects [22]. Clinical characteristics and motor findings of these patients have been previously reported [23]. Measures of motor speed included finger tapping, timed gait, and timed reading of a standard passage (the Grandfather Passage).

\section{Neuropsychological Testing}

The cognitive testing battery consisted of the Dementia Rating Scale (DRS-2) from Mattis and colleagues [24] and the Delis-Kaplan Executive Function System (D-KEFS) [25]. The scoring of these tests is normed for age and educational level. The behavioral and mood testing battery consisted of the Beck Depression Inventory (BDI-2) [26], the Frontal Systems Behavioral Scale (FrSBe) [27], and the UCLA Neuropsychiatric Index (NPI) [28]. Behavioral ratings by the caregivers were used for the FrSBe and the NPI. When patients were unable to carry out a subtest in the DRS- 2 and D-KEFS because of limited motor function, that subtest was not included in the patient's individual assessment. However, every subtest was completed by at least 20 patients in each group. Patients were classified as ALSci $[3,6]$ if they scored in the lowest 5 th percentile on at least two of the subscores of the D-KEFS and the low scores could not be accounted for by motor or speech dysfunction. Patients were classified as ALSbi $[3,6]$ if they exhibited at least two supportive diagnostic features with clinical history and supportive testing using the FrSBe and NPI. ALS and PLS patients who completed sufficient subtests of the D-KEFS battery, the NPI, and FrSBe underwent imaging studies.

\section{Principal Component Analysis}

To reduce the dimensions of the cognitive testing scores for comparison with imaging measures, a principal components analysis (PCA) of the D-KEFS was conducted, similar to that done in a prior study of FTLD patients [13]. Eleven D-KEFS subscores that were completed by 43 of the 50 patients were used as variables in the PCA (table 1; online suppl. table 1, www. karger.com/doi/10.1159/000353456). Although most D-KEFS tests are carried out under timed conditions, test scores that measured motor speed without content (e.g. Tower test mean time to first move, Trail motor speed) were excluded (table 2). The Kaiser-Meyer-Olkin measure (0.659) indicated the sample size was adequate and Bartlett's test of sphericity ( $\mathrm{p}<$ 0.001 ) indicated the variables were appropriate for PCA. PCA with varimax rotation revealed 3 components with eigenvalues $>1.0$, cumulatively accounting for $72.6 \%$ of the variance. The first component (PC1) accounted for about one third of the variance. PC1 loaded most heavily on the fluency subscores (table 1 , loading $>0.7$ in bold). The second component (PC2) was most heavily weighted on the sorting subscores. The third component (PC3) was heavily weighted on the subscores of the Trails battery. For simplicity, throughout the rest of this article, the three principal components will be referred to as 'fluency', 'sorting', and 'Trails'.

\section{Imaging Acquisition}

Magnetic resonance imaging (MRI) studies were performed on a 3-tesla scanner (Philips Achieva, Best, The Netherlands) using a receive-only, 8-channel SENSE head coil. For volu- 
Dementia

and Geriatric

Cognitive Disorders

Table 1. D-KEFS test scores used as variables in the PCA with loading for each variable

\begin{tabular}{l|l}
\hline Dement Geriatr Cogn Disord Extra 2013;3:233-250 \\
\hline DOI: $10.1159 / 000353456$ & $\begin{array}{l}\text { C) 2013 S. Karger AG, Basel } \\
\text { www.karger.com/dee }\end{array}$ \\
\hline
\end{tabular}

Meoded et al.: Imaging Findings Associated with Cognitive Performance in Primary Lateral Sclerosis and Amyotrophic Lateral Sclerosis

\begin{tabular}{|c|c|c|c|}
\hline & \multicolumn{3}{|c|}{ Principal component } \\
\hline & $\begin{array}{l}1 \\
\text { fluency }\end{array}$ & $\begin{array}{l}2 \\
\text { sorting }\end{array}$ & $\begin{array}{l}3 \\
\text { trails }\end{array}$ \\
\hline \multicolumn{4}{|l|}{ Sorting } \\
\hline Free sort confirmed correct & 0.163 & 0.909 & 0.098 \\
\hline Free sort description & 0.245 & 0.904 & 0.072 \\
\hline \multicolumn{4}{|l|}{ Tower test } \\
\hline Total achievement score & 0.182 & 0.424 & -0.633 \\
\hline \multicolumn{4}{|l|}{ Trails } \\
\hline Visual scan & 0.339 & 0.1 & 0.817 \\
\hline Number sequence & 0.063 & 0.346 & 0.734 \\
\hline Letter sequence & 0.377 & 0.513 & 0.495 \\
\hline Number-letter switching & 0.597 & 0.119 & 0.350 \\
\hline \multicolumn{4}{|l|}{ Fluency } \\
\hline Letter fluency & 0.726 & 0.252 & 0.215 \\
\hline Category fluency & 0.733 & 0.337 & 0.287 \\
\hline Correct response & 0.909 & 0.146 & -0.014 \\
\hline Switch accuracy & 0.887 & 0.097 & -0.112 \\
\hline
\end{tabular}

Scores with the strongest loading on each component are indicated in bold type.

metric and thickness measurements, a high-resolution T1-weighted image was obtained using a three-dimensional turbo field echo sequence: $\mathrm{TR}=8.6 \mathrm{~ms}, \mathrm{TE}=3.9, \mathrm{TI}=700$, flip angle $=6^{\circ}, \mathrm{FOV}=240 \mathrm{~mm}$, matrix size $=256 \times 256$, slice thickness $=1 \mathrm{~mm}$, and 140 axial slices . In addition, multislice diffusion-weighted imaging was acquired using a single-shot spin-echo echo-planar sequence: $\mathrm{TE}=86 \mathrm{~ms}, \mathrm{FOV}=240 \mathrm{~mm}$, matrix size $=96 \times 96$ reconstructed to 128 $\times 128$ (voxel size $=1.875 \times 1.875 \times 2.5 \mathrm{~mm}$ ), slice thickness $=2.5 \mathrm{~mm}$, and 55 contiguous axial slices aligned parallel to the AC-PC line. Diffusion weighting was performed along 32 noncollinear directions with $\mathrm{a} b$ value of $1,000 \mathrm{~s} / \mathrm{mm}^{2}$, and a single volume was collected with no diffusion gradients applied (b0). The diffusion sequence was repeated 4 times to increase the signal-to-noise ratio. The scan time for each diffusion sequence was $4 \mathrm{~min}$.

\section{Atlas-Based Analysis of DTI}

Semiautomated computerized atlas-based analysis was used to segment and label white matter tracts. DTI postprocessing was performed off-line using MRI Studio software (H. Jiang and S. Mori, Johns Hopkins University, www.MriStudio.org) [29]. The raw diffusion-weighted images were first coregistered to one of the least diffusion-weighted images and corrected for eddy current and subject motion using a 12-mode affine transformation with Automated Image Registration [30,31]. The six elements of the diffusion tensor, the fractional anisotropy (FA), the eigenvectors, and the eigenvalues were calculated for each voxel with multivariate linear fitting $[32,33]$. Before the normalization procedure, the skull was stripped using the diffusion-weighted images and a skull-strip tool in the RoiEditor software. The images were then normalized to the ICBM-DTI-81 coordinates [34] using a 12-mode affine transformation with DiffeoMap. Subsequently, a nonlinear transformation using dual-contrast large deformation diffeomorphic metric mapping was applied to register the FA and Trace images to the 1 -mm isotropic resolution template $(181 \times 217 \times 181$ matrix $)$. Because both linear and nonlinear transformations are reciprocal procedures, the inversely transformed WMPMII parcellation map was superimposed onto the original DTI metric maps from the patient leading to the parcellation of the brain into 130 anatomical structures, including gray matter 
Table 2. Scaled scores on the D-KEFS battery in ALS and PLS patients

\begin{tabular}{|c|c|c|c|c|}
\hline & \multicolumn{2}{|l|}{ ALS } & \multicolumn{2}{|l|}{ PLS } \\
\hline & mean $\pm S D$ & $\mathrm{n}$ & mean $\pm \mathrm{SD}$ & $\mathrm{n}$ \\
\hline \multicolumn{5}{|l|}{ Sorting } \\
\hline Free sort confirmed correct & $10.95 \pm 3.19$ & 20 & $10.39 \pm 3.00$ & 23 \\
\hline Free sort description & $11.15 \pm 3.00$ & 20 & $10.57 \pm 2.76$ & 23 \\
\hline Sort recognition description & $9.73 \pm 3.23$ & 11 & $9.00 \pm 3.56$ & 21 \\
\hline Total achieve score & $10.40 \pm 2.52$ & 20 & $10.63 \pm 2.34$ & 24 \\
\hline \multicolumn{5}{|l|}{ Tower test } \\
\hline Total achievement & $10.40 \pm 2.52$ & 20 & $10.63 \pm 2.34$ & 24 \\
\hline Mean first move time & $12.24 \pm 3.51$ & 17 & $13.64 \pm 2.72$ & 22 \\
\hline Time per move ratio & $6.32 \pm 3.59$ & 19 & $7.91 \pm 2.70$ & 23 \\
\hline Move accuracy ratio & $10.59 \pm 1.50$ & 17 & $9.64 \pm 1.50$ & 22 \\
\hline Rule violations per item ratio & $9.82 \pm 2.65$ & 17 & $10.59 \pm 0.59$ & 22 \\
\hline \multicolumn{5}{|l|}{ Trail making } \\
\hline Visual scanning & $9.00 \pm 3.54$ & 21 & $7.48 \pm 2.64$ & 23 \\
\hline Number sequencing & $9.57 \pm 3.14$ & 21 & $9.39 \pm 3.43$ & 23 \\
\hline Letter sequencing & $9.62 \pm 3.79$ & 21 & $10.09 \pm 2.92$ & 23 \\
\hline Number-letter switching & $9.57 \pm 4.02$ & 21 & $10.30 \pm 2.79$ & 23 \\
\hline Motor speed & $9.00 \pm 3.73$ & 21 & $9.30 \pm 2.10$ & 23 \\
\hline \multicolumn{5}{|l|}{ Twenty questions } \\
\hline Initial abstraction & $10.40 \pm 3.65$ & 20 & $9.77 \pm 2.67$ & 22 \\
\hline Total questions asked & $10.95 \pm 3.25$ & 20 & $10.36 \pm 3.39$ & 22 \\
\hline Total weighted achievement & $10.65 \pm 3.72$ & 20 & $10.64 \pm 3.30$ & 22 \\
\hline \multicolumn{5}{|l|}{ Verbal fluency } \\
\hline Letter fluency & $9.86 \pm 3.60$ & 21 & $10.05 \pm 3.34$ & 22 \\
\hline Category fluency & $10.52 \pm 4.93$ & 21 & $9.95 \pm 2.38$ & 22 \\
\hline Correct responses & $11.67 \pm 3.67$ & 21 & $9.86 \pm 3.00$ & 22 \\
\hline Switch accuracy & $11.76 \pm 2.86$ & 21 & $10.14 \pm 2.49$ & 22 \\
\hline \multicolumn{5}{|l|}{ Word context } \\
\hline Total consecutive correct & $10.84 \pm 3.59$ & 19 & $10.48 \pm 2.61$ & 23 \\
\hline \multicolumn{5}{|l|}{ Proverbs } \\
\hline Free answer & $10.79 \pm 4.08$ & 19 & $10.74 \pm 2.12$ & 23 \\
\hline
\end{tabular}

Scaled scores are normed for age and education. Normal mean $=10 ; \mathrm{SD}=3$. Subscores used as variables in the PCA are in bold.

regions and white matter tracts. One further step in the segmentation of the white matter consisted of thresholding all the gray matter and cerebrospinal fluid by excluding regions of interest of FA $<0.2$ and Trace $>0.0045$ on the corresponding images. The quantitative analysis was carried out on the FA, Trace, axial diffusivity (AD), and radial diffusivity (RD) in white matter structures parcellated in the atlas-based analysis [35].

Eleven white matter association area tracts on each side were chosen for analysis (fig. 1), including the uncinate fasciculus, superior longitudinal fasciculus (SLF), superior frontooccipital fasciculus (SFO), inferior fronto-occipital fasciculus (IFO), posterior thalamic radiation (PTR), and the fornix. The corpus callosum was segmented into three portions: the genu, body, and splenium. The cingulum was segmented into the portion underlying the cingulate cortex and a hippocampal portion. These association tracts were chosen because of literature suggesting their association with the cognitive tests used, or because of their involvement in ALS or FTLD. The corticospinal tract was not included in the analysis, as the changes associated with ALS and PLS diagnoses and motor function have been previously reported for these patients [23]. 
Meoded et al.: Imaging Findings Associated with Cognitive Performance in Primary Lateral Sclerosis and Amyotrophic Lateral Sclerosis

Fig. 1. FA maps with color overlays showing the white matter tracts included in the analysis. Tracts were segmented by atlas analysis. The evaluated tracts were the uncinate fasciculus (UNC), SLF, SFO, IFO, PTR, and the fornix (Fx). The corpus callosum was segmented into three portions: the genu, body, and splenium (GCC, BCC, and SCC). The cingulum was segmented into the portion underlying the cingulate cortex (CGC) and a parahippocampal portion (CGH).

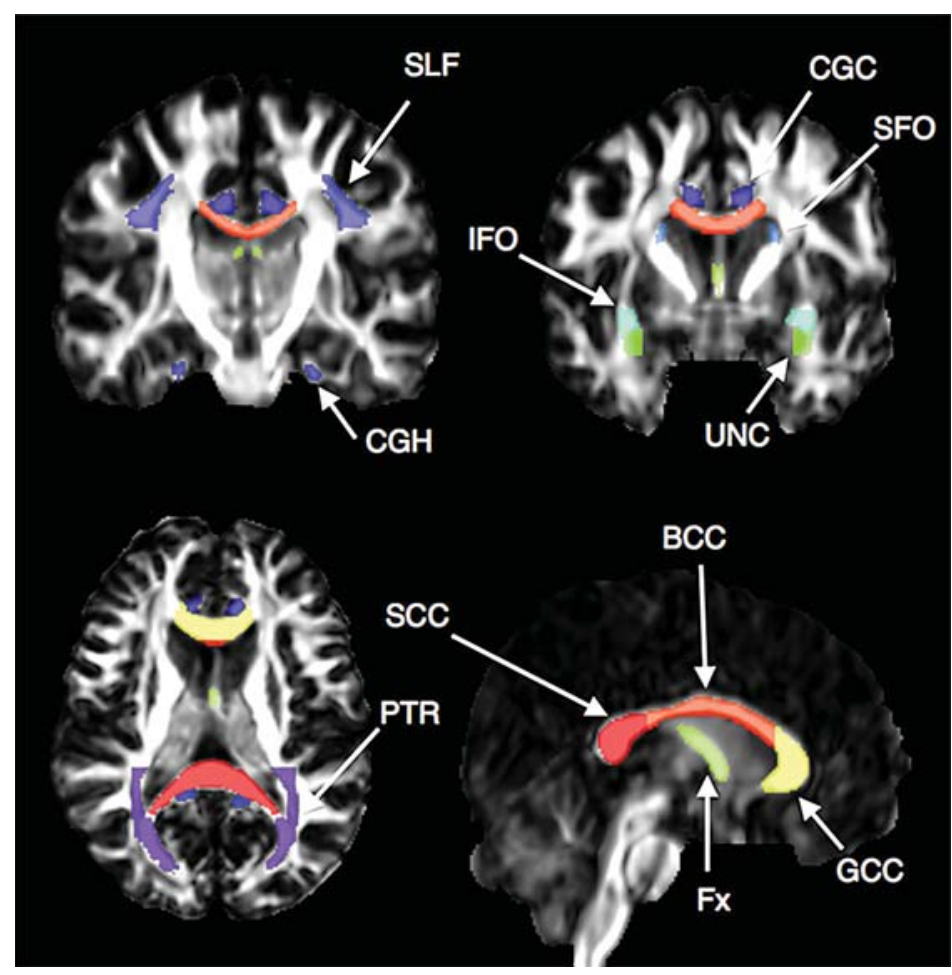

\section{Volumetric Analysis}

Statistical parametric mapping was used to assess volumetric differences between patients and healthy controls and the association between volumetric differences and cognitive measures. Voxel-based morphometry (VBM) analysis of the data was performed with SPM8 (http://www.fil.ion.ucl.ac.uk/spm/software/spm8) for Matlab version 2012a. Images were registered and segmented using the new segment and DARTEL (diffeomorphic anatomical registration through exponentiated lie algebra) algorithm [36], normalized to the Montreal Neurological Institute (MNI) space, and smoothed with a 10-mm FWHM (full width at half maximum) filter. These preprocessing steps were carried out according to the principles outlined by Ridgway et al. [37]. We were interested in evaluating the gray matter volumetric changes of patients affected by ALS and patients with PLS compared to healthy subjects. Thus, both groups were analyzed separately. The preprocessed (i.e. segmented, modulated, normalized, and smoothed) scans from each patient group were compared to the scans from the group of age-matched healthy control subjects using a two-sample t test. For the statistical analysis, an explicit absolute threshold mask of 0.05 was used. Age, gender, and total intracranial volume were entered as covariates of no interest. Finally, in all our analyses we used a 0.05 family-wise error threshold [38].

Subsequent association analyses were limited to areas of significant gray matter volume reduction. The relationship between voxel values and each of the three scores from the D-KEFS PCA and the DRS- 2 total and memory scores was examined using one-tailed tests (one for each component), assuming that decreased performance in these tests would be associated with decreased gray matter volume; however, we also tested the opposite relationship as recommended when performing VBM studies. We used areas of significant gray matter volume reduction derived from the first-step analysis as an explicit mask. An uncorrected statistical threshold of $\mathrm{p}<0.001$ was applied with a 30 -voxel threshold. In addition, age, gender, and total intracranial volume were entered in the model as covariates of no 
interest. Identification of brain regions and corresponding Brodmann areas were determined using PickAtlas software [39].

\section{Statistical Analysis}

Data are presented as means \pm SD. Statistical analyses were performed using SPSS Statistics version 17.0 and SAS 9.2. The normality of clinical and demographic data was confirmed with the Kolmogorov-Smirnov test, and a one-way ANOVA with unequal variance was used to compare groups. Fisher's exact test was used to test differences in the proportions of ALS and PLS patients with abnormal raw total scores on the DRS-2 and in the proportion of patients classified as ALSci and ALSbi.

A two-step statistical model was used to examine the relationship between diffusion properties of the selected white matter association tracts and cognitive measures. In the first step, the variables of age, gender, disease duration, ALSFRS-R score, diagnosis, and time to read a standard passage were evaluated as covariates, based on a significance level of 0.1 . In the second step, a regression analysis was performed with a linear model including the selected covariate(s), the diffusion metrics and their interactions (which were dropped from the model if $p>0.1$ ), and the cognitive measures. The cognitive measures evaluated were the DRS- 2 total score, DRS-2 memory subscore, three D-KEFS principal components, and the D-KEFS scaled subscores for free sort description, fluency switch accuracy, and letter fluency. The first two scaled subscores were included to assess measures independent of motor ability. Letter fluency is a timed test likely to be affected by rate of speech. Differences in diffusion properties between the patients with and without mild cognitive or behavioral impairment (ALSci, ALSbi) were evaluated with a similar regression analysis model with age, gender, disease duration, and ALSFRS-R score considered as covariates. The diffusion properties analyzed for the 11 tracts on each side were FA, RD, and AD. Due to the large number of variables and the exploratory nature of this analysis, an uncorrected $p=0.01$ was used as the significance level.

\section{Results}

Age, gender, and years of education did not differ significantly between groups (table 3). As expected, because PLS is defined as having at least 4 years of progressive spasticity [40], disease duration was shorter in ALS patients than in PLS patients $(\mathrm{p}<0.001)$. The ALSFRS-R, a measure of function and strength, did not differ between the ALS and PLS patient groups. Dexterity was not different between PLS and ALS patients, as measured by speed of finger tapping (table 3), and there were no differences in the rate of speech as measured by the time needed to read a standard passage.

Neuropsychological testing was carried out on all patients, although not every patient was able to do every test in the battery (table 2). Not all were able to undergo neuroimaging studies, typically because the severity of their illness limited tolerance or they had implanted medication pumps. Technically adequate studies were obtained for volumetric analysis from 13 ALS and 17 PLS patients, and for DTI analysis from 13 ALS and 15 PLS patients. Of the patients who underwent MRI, 6 patients (1 PLS, 5 ALS) had mild cognitive impairment fulfilling the criteria for ALSci and 8 patients ( 3 PLS, 5 ALS) had mild behavioral impairment fulfilling the criteria for ALSbi. There was no difference in motor measures of finger tapping or speech between the group of patients with ALSci and ALSbi and those without cognitive or behavioral impairment. The demographics of the subset of patients who were able to complete the imaging studies did not differ from those of the study population as a whole (table 3 ). The 17 healthy controls who underwent MRI were matched for age and gender, and had normal neurological examinations but not formal neuropsychological testing. 
Table 3. Characteristics of the patient and control groups, and subsets participating in imaging studies

\begin{tabular}{|c|c|c|c|c|c|c|c|c|}
\hline & \multicolumn{2}{|c|}{$\begin{array}{l}\text { Neuropsychological } \\
\text { testing (all patients) }\end{array}$} & \multicolumn{3}{|l|}{ DTI subset $^{1}$} & \multicolumn{3}{|c|}{ VBM subset } \\
\hline & PLS & ALS & PLS & ALS & $\begin{array}{l}\text { healthy } \\
\text { controls }\end{array}$ & PLS & ALS & $\begin{array}{l}\text { healthy } \\
\text { controls }\end{array}$ \\
\hline Male:female, $\mathrm{n}$ & $13: 12$ & $12: 13$ & $6: 9$ & $5: 8$ & $8: 7$ & $7: 10$ & $5: 8$ & $9: 8$ \\
\hline Age, years & $56.3 \pm 7.9$ & $57.7 \pm 11.2$ & $57.6 \pm 7.6$ & $51.0 \pm 8.3$ & $59.5 \pm 6.0$ & $58.9 \pm 8.0$ & $51.0 \pm 8.3$ & $59.2 \pm 5.8$ \\
\hline Education, years & $15.8 \pm 2.6$ & $14.8 \pm 2.6$ & $15.8 \pm 2.9$ & $14.7 \pm 2.8$ & & $15.7 \pm 2.8$ & $14.7 \pm 2.8$ & \\
\hline Disease duration, years & $11.7 \pm 6.5^{*}$ & $2.3 \pm 1.4$ & $12.0 \pm 6.5^{*}$ & $2.5 \pm 1.5$ & & $11.6 \pm 6.2^{*}$ & $2.5 \pm 1.5$ & \\
\hline ALSFRS-R score & $38.8 \pm 4.5$ & $36.2 \pm 6.6$ & $36.7 \pm 4.4$ & $35.5 \pm 4.3$ & $48.0 \pm 0.0$ & $36.9 \pm 4.2$ & $35.5 \pm 4.3$ & \\
\hline Timed reading (standard passage), s & $75.6 \pm 23.6$ & $75.3 \pm 23.4$ & $65 \pm 17.6$ & $70.1 \pm 15.5$ & & $74.4 \pm 20.2$ & $70.1 \pm 15.5$ & \\
\hline Right finger tap/15 s, n & $49.1 \pm 15.6$ & $56.7 \pm 27.9$ & $52.8 \pm 15.2$ & $54.6 \pm 30.6$ & & $51.1 \pm 15.2$ & $54.6 \pm 30.6$ & \\
\hline Left finger tap/15 s, n & $42.9 \pm 14.1$ & $54.5 \pm 25.1$ & $45.2 \pm 15.7$ & $51.4 \pm 24.8$ & & $44.3 \pm 14.9$ & $51.4 \pm 24.8$ & \\
\hline
\end{tabular}

Values represent means $\pm \mathrm{SD}$, except for the male:female ratio. ${ }^{*} \mathrm{p}<0.05$ vs. ALS. ${ }^{1}$ Studies with atlas-based analysis.

\section{Neuropsychological Studies}

Twenty-three ALS and 23 PLS patients completed all portions of the DRS-2. The proportion of patients who scored below normal (DRS-2 raw score <133) was higher in the ALS patient group (35\%) than in the PLS patient group (5\%; $p=0.022$, Fisher's exact test; fig. 2a). The lower scores occurred across all five subscores of the DRS-2, including those, such as conceptualization, that do not rely on motor function (ANOVA F $=17.04, \mathrm{p}<0.001$; fig. $2 \mathrm{~b}$ ). No patient met the criteria for FTLD of Neary et al. [4].

Table 2 shows the number of patients completing each test in the D-KEFS battery. There was no difference between the PLS and ALS patient groups in the mean scaled scores for any D-KEFS subtest (table 2). However, 9 ALS and 2 PLS patients individually met the criteria for ALSci, with two or more scaled scores on subtests of the D-KEFS that were at or below the 5th percentile. The proportion of patients with ALSci was higher in the ALS patient group than the PLS patient group ( $p=0.021$, Fisher's exact test). As a group, patients meeting the ALSci criteria had lower scores on multiple subtests of the D-KEFS compared to patients without ALSci. As illustrated in figure $2 \mathrm{c}$ and d, patients with cognitive impairment (gray bars) scored lower on tests in which performance is less dependent on motor function (sort description, fluency switch accuracy), as well as on tests more affected by motor function (free sort description, letter fluency). The classification of ALSci was based on patient scores on the D-KEFS test battery, but it is notable that the ALSci group included all patients who had abnormal raw scores on the DRS-2.

Six ALS patients and 5 PLS patients fulfilled the criteria for ALSbi, including 3 ALS patients and 1 PLS patient who met the criteria for both ALSci and ALSbi. Apathy and disinhibition were the most common behavioral abnormalities described by caregivers on the FrSBe questionnaire. Of note, however, 4 of the patients classified as ALSbi also scored in the mildmoderately depressed range and 1 in the severely depressed range on the BDI-2, which is consistent with our earlier study on the frequency of depression in ALS and PLS patients [41]. There was no significant difference between patients with and without ALSci/bi in the ALSFRS-R or measures of movement speed (tapping, speech) in the PLS and ALS groups.

\section{DTI and Performance on Cognitive Tests}

Gender, disease duration, and diagnosis (PLS vs. ALS) were not significant covariates and were dropped from the regression model. The D-KEFS letter fluency score was adjusted for the covariate of timed reading. Significant interactions of age and the ALSFRS-R score oc- 


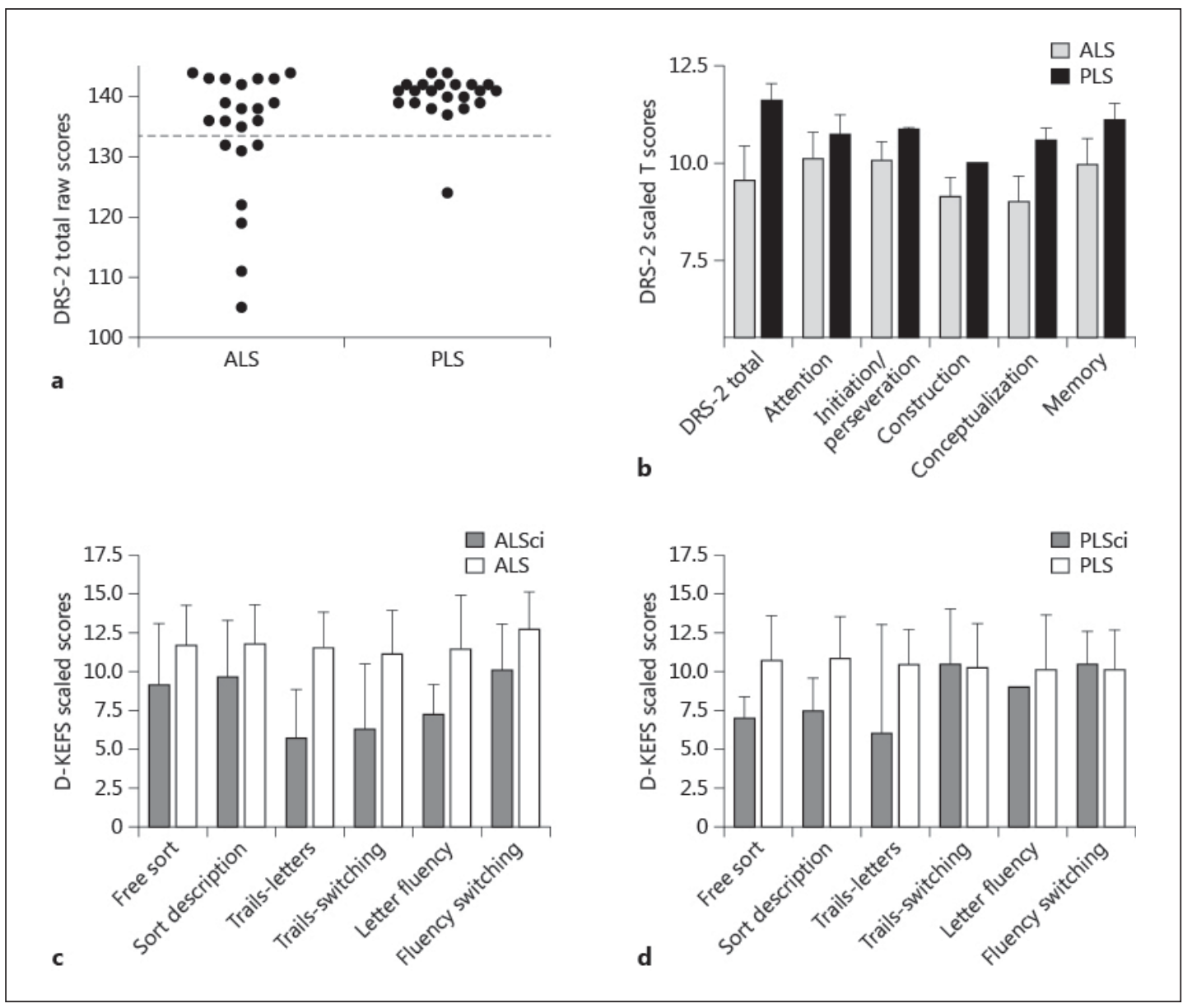

Fig. 2. Cognitive test scores in ALS and PLS patients. a DRS-2 total raw scores for each patient. Values above 133 (dashed line) are considered normal according to reference values. b Scaled scores for the five domains of the DRS-2. Group means \pm SD are shown. The DRS-2 scaled scores were significantly less in ALS patients than in PLS patients (ANOVA, $\mathrm{p}<0.001$ ). c, d Scaled scores on selected D-KEFS subtests for ALS patients (c) and PLS patients (d) fulfilling the consensus criteria for cognitive impairment (ALSci) compared to patients without cognitive impairment (means \pm SD).

curred with the DRS-2 score, and of the ALSFRS-R score with the sorting principal component. Significant covariate interactions were included in the regression model.

Patients were pooled to examine the relationship between imaging and cognitive measures because diagnosis was not a significant covariate. Diffusion metrics of several white matter tracts were found to be associated with performance on the DRS-2 and the D-KEFS (table 4). The total DRS-2 score was associated with AD of the body of the corpus callosum and with AD and RD of the right SFO (fig. 3b, c). The DRS-2 memory subscore was associated with AD of the IFO bilaterally (fig. 4a) and reductions in FA in the right PTRs (fig. 4b). The fluency principal component was associated with AD of the left SLF (fig. 3f). The scaled subscores for the fluency 'correct response' and switch accuracy were associated with AD in the cingulate bundle in the portions extending into the temporal lobe overlying the left hippocampus (fig. 4c, d). The letter fluency scaled score was associated with RD in the PTRs (fig. 4b). The sorting principal component was associated with $\mathrm{AD}$ of the splenium of the callosum (fig. 3d, 4c). 




Fig. 3. Sagittal (a) and axial (b-f) views of FA maps with color overlays of white matter structures with significant associations between DTI metrics and neuropsychological tests as listed in table 4. a Axial slices are shown in radiological convention with the left brain on the right, body of the corpus callosum (BCC). b, c SFO. d Splenium of the corpus callosum (SCC). e PTRs. f SLF. Scale denotes uncorrected $p$ value, and thresholds below 0.01 are shown.

Table 4. Association of neuropsychological test performance with diffusion properties of white matter tracts

\begin{tabular}{llllll}
\hline Neuropsychological test & White matter tract & Side & $\begin{array}{l}\text { DTI } \\
\text { metric }\end{array}$ & $\beta$ & p value \\
& & & & & \\
DRS-2 & & R & AD & 333.83 & 0.00484 \\
$\quad$ Total score & corpus callosum-body & R & AD & 339.85 & 0.00005 \\
Total score & SFO & R & RD & 274.92 & 0.00480 \\
Total score & SFO & L & AD & 30.64 & 0.00706 \\
Memory score & IFO & R & AD & 26.46 & 0.00718 \\
Memory score & IFO & R & FA & -0.07 & 0.00554 \\
Memory score & PTR & & & & \\
D-KEFS principal components & & L & AD & 15.49 & 0.00871 \\
Fluency (PC1) & SLF & L & AD & 114.56 & 0.00276 \\
Sorting (PC2) & corpus callosum-splenium & & & & \\
D-KEFS scaled scores & & R & RD & 52.71 & 0.00742 \\
Letter fluency & PTR & L & AD & 34.47 & 0.00556 \\
Fluency correct response & cingulum-hippocampal portion & L & AD & 34.89 & 0.00760 \\
Fluency correct response & corpus callosum-splenium & L & AD \\
Fluency switch accuracy & cingulum-hippocampal portion & L & AD & 27.69 & 0.00799 \\
\hline
\end{tabular}




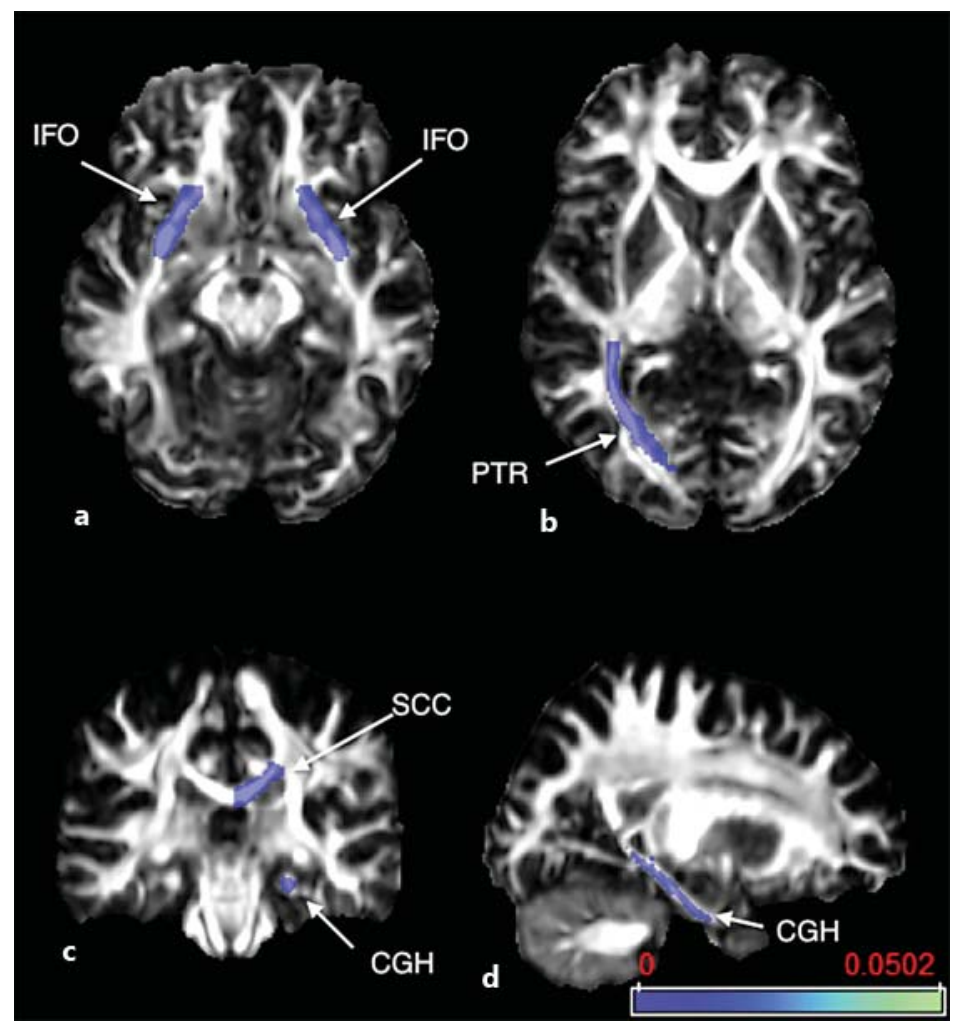

Fig. 4. Axial (a, b), coronal (c), and sagittal (d) views of FA maps with color overlays of white matter structures with significant associations between DTI metrics and particular neuropsychological tests as listed in table 4. The DRS-2 memory subscore was associated with AD of the IFO bilaterally (a), and the right PTR was associated with the memory score and letter fluency (b). Other fluency subscores were associated with diffusion properties of the splenium of the corpus callosum (SCC) (c) and the parahippocampal portion of the left cingulum (CGH) (d). Scale denotes uncorrected p value, and thresholds below 0.01 are shown.

\section{DTI and ALSci and ALSbi}

After adjusting for covariates there were no significant DTI findings associated with ALSci $(p>0.01)$. In patients with ALSbi, higher FA was seen in the left cingulum ( $p=0.0049)$ and lower RD in the right cingulum white matter $(\mathrm{p}=0.0087)$. The multivariate analysis showed an interaction between ALSbi and the ALSFRS-R score covariate within two white matter tracts: there was no association with the ALSFRS-R score in patients with ALSbi; however, in behaviorally normal patients, higher ALSFRS-R scores were associated with greater AD in the left PTR $\left(R^{2}=0.44, p=0.0014\right)$ and a trend toward $R D$ in the $\operatorname{SLF}\left(R^{2}=0.237\right.$, $\mathrm{p}=0.029$ ).

\section{VBM Analysis}

Compared to healthy subjects, ALS patients had one cluster $(k=109, p=0.012$, familywise error corrected) with reduced gray matter volume in the right perirolandic area, and the MNI coordinates of the peak voxels were $28,-33$, and 45 (fig. 5a). PLS patients had reduced gray matter volume in one cluster (fig. $5 ; \mathrm{k}=1126, \mathrm{p}=0.027$, family-wise error corrected) in the region of the right motor cortex, and the MNI coordinates of peak voxels were $31,-25$, and 36 . There were no significant associations between these areas of reduced gray matter and performance on any neuropsychological testing. In addition, no volumetric differences were seen between patients with and without ALSci/bi. 
Fig. 5. Volumetric differences between patients and healthy controls. Axial T1-weighted images with overlaid results of VBM analysis show a cluster with reduced gray matter volume in the right perirolandic area in ALS patients (a) and a cluster with reduced gray matter volume in the right motor cortex in PLS patients (b).
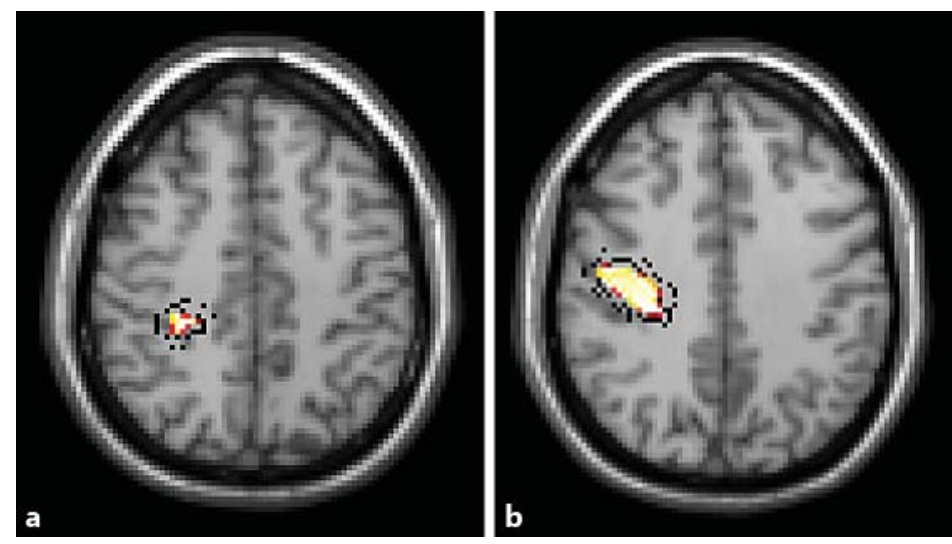

\section{Discussion}

Overall, the cognitive performance of many of our patients was consistent with a subtle executive dysfunction, although none of the patients met the criteria for FTLD at the time of evaluation. About one third of the ALS patients in this study were found to have mild cognitive deficits, a proportion that is similar to several previous reports $[1,3,42]$. We found that a much lower proportion of PLS patients, 14\%, met criteria for either cognitive or behavioral impairment. This lower proportion could not be accounted for by differences in motor function, which did not differ between the ALS and PLS patient groups or between patients who met the criteria for ALSci and those who did not. Patients who met the criteria for ALSci scored lower across a broad range of cognitive tests, including those that were not dependent on motor function, such as the description of the rules used in a sorting task. Although only a handful of studies have measured cognitive function in PLS patients, there are widely varying estimates of its occurrence. One retrospective review of clinic patients reported that the majority of PLS patients had cognitive impairment [11]. However, it has also been reported that $39 \%$ of PLS patients had cognitive impairment [12], and another study even found that only $22 \%$ of PLS patients had cognitive impairment [43]. The proportion of PLS patients with cognitive impairment was somewhat lower in our study than in these earlier reports. This difference may result from different case definitions for PLS that were used in each study or from a bias toward higher functioning patients in our study, which was limited to patients who could travel to our institution.

The second aim of our study was to explore the relationship between performance on neuropsychological tests and imaging measures. The relationship between impaired performance in specific cognitive domains and damage to specific brain structures can provide insight into the cerebral changes underlying cognitive presentations in ALS and PLS patients. Although several studies have examined the relationship between cognitive impairment and neuroimaging findings in ALS patients [18, 19,44,45], to our knowledge there have not been similar studies in PLS patients. With VBM, we found atrophy in gray matter regions predominantly in the motor and perirolandic cortices. These reductions were not correlated with performance on cognitive tests. In contrast, DTI revealed changes in several white matter tracts connecting extramotor regions of the brain that were related to patient performance on neuropsychological tests. The DTI analysis focused on 11 association or commissural white matter tracts, which were selected based on literature descriptions indicating their involvement in ALS or in executive function [18, 19, 46].

In the white matter tracts, we measured the $\mathrm{AD}, \mathrm{RD}$, and FA. FA maps spatially localize the degree of anisotropic diffusion, which is believed to be related to the degree of axonal 
packing and myelination. Axonal disruption produces decreased FA. In tracts containing a mixture of fibers with differing orientations, FA can theoretically become increased due to either reduced axonal branching or reduced integrity of crossing fibers, leading to an increased net directional preference of water diffusion within each voxel. AD, the eigenvalue of the primary eigenvector, is also predominantly modified by acute axonal damage, which causes fragmentation of axons and barriers to the longitudinal diffusion of water molecules. RD, the mean of the second and third eigenvalues, may preferentially reflect myelin injury or increased astrogliosis [47].

Changes in diffusion properties related to cognitive test scores mostly occurred in long white matter tracts connecting the frontal lobes to the more posterior cortical regions. One caveat is that axons traveling to different destinations can be contained within these tracts. For example, the SLF contains longer fibers connecting the frontal cortex with the dorsolateral parietal and temporal cortices as well as shorter U-shaped fibers connecting the fronto-parietal, parieto-occipital, and parieto-temporal cortices [48]. Because the atlas-based method used in this study defines white matter tracts based on anatomical locations, it does not distinguish between subsets of fibers with different destinations within a tract and may underestimate those changes that affect only a portion of the tract.

Scores on the DRS- 2 were associated with diffusivity measures of the SFO and IFO which, together with the SLF and the uncinate fasciculus, make up the longitudinal association tracts connecting frontal regions to the parietal, temporal, and occipital lobes. Although the total DRS-2 score was associated with right hemisphere tracts, the DRS-2 memory subscore, which includes both verbal and visual memory items, was related to diffusivity in the IFO bilaterally. The IFO connects regions of the frontal lobe with temporal and occipital cortices. The left IFO has been implicated as being important for semantic memory in healthy adults [49] and was found to exhibit reduced FA and increased diffusivity in patients with progressive supranuclear palsy with dysexecutive problems, apathy, and personality change [50]. In ALS patients, lower FA values of the IFO were reported to be correlated with worse performance in the Wisconsin Card Sorting Test [19], which requires working memory for visual stimuli.

Because the Delis-Kaplan battery that was used to test executive function provides multiple subscores within nine separate tests, we carried out a PCA to reduce the number of variables and emphasize those less likely to be affected by motor impairment. Three components were identified that could account for most of the variance, similar to other studies of the D-KEFS factor structure that found a 3-factor solution using different methods [51]. The first principal component, which we termed fluency (PC1), was heavily loaded on fluency subscores, with a smaller contribution from switching subscores of the Trails test. Our fluency component has a similar composition to the 'monitoring' factor identified in an earlier study [51]. The variables comprising the fluency component require categorization, manipulating content in working memory, and cognitive flexibility. The fluency component was associated with diffusivity in the left SLF, suggesting these tasks require the integrity of tracts connecting the left frontal lobes with the parietal, occipital, and temporal regions. This is consistent with atrophy of the prefrontal and posterior parietal cortex (regions connected through the SLF) and correlated with a loss of set shifting in patients with various neurodegenerative diseases [52]. Among the individual subscores of the D-KEFS fluency test, the correct response score and switch accuracy score were associated with the left parahippocampal portion of the cingulum, connecting the frontal, precuneus, and temporal cortices. Diffusivity changes in the anterior and posterior cingulum have been associated with declining memory and attention in older adults without dementia [53], and the cingulum is thought to be important for connectivity of the precuneus/posterior cingulate cortex and medial frontal cortex in the default network in resting fMRI [54]. The cingulum has been implicated in working memory and cognitive control in patients with mild cognitive impairment [55]. 
Most of these findings are consistent with the traditional view that verbal fluency reflects left frontal function [56], as well as a neuropathological study that identified damage to the SLF in six ALS brains examined [57]. fMRI studies have shown that patients with verbal fluency deficits have impaired activation in networks implicated in letter fluency and confrontation naming, which include the dorsolateral prefrontal cortex, anterior cingulum, inferior frontal gyrus, and regions of the occipito-temporal pathways [58]. Primate studies support the role of the SLF in connecting these areas. In primates, the SLF has been shown to have three distinct branches: SLF I connects the superior frontal gyrus to the posterior medial and caudal superior parietal lobule, SLF II originates from the dorsolateral prefrontal cortex and ends in the occipito-parietal area and the caudal inferior parietal lobule, and SLF III links the posterior part of the inferior frontal gyrus to the rostral portion of the inferior parietal lobule [59]. Damage to SLF II may account for changes in our patients since it connects specific anatomical regions affected in our population.

The second component of the D-KEFS, termed sorting (PC2) requires concept formation and the ability to deduce categories. Sorting (PC2) was associated with AD in the splenium of the corpus callosum. The free sort description subscore itself was not associated with changes in the diffusion metrics of the white matter tracts examined. This finding is somewhat at variance with Fine et al. [60], who also used the D-KEFS and found that free sort description subscores were associated with reduced frontal volumes in patients with a variety of neurodegenerative disease with cognitive impairment. Libon et al. [20] also reported that performance on sort recognition scores was correlated with left prefrontal and parietal cortical thinning in 16 ALS patients divided into three groups based on performance in the sorting task. The lack of cortical atrophy could reflect a lesser degree of cognitive dysfunction in our patients. Diffusion changes in the splenium also differ from Sarro et al. [19], who reported that the Wisconsin Card Sort Test correlated with changes in diffusion properties of the IFO and ILF bilaterally in ALS. These differences may reflect limitations of the small sample sizes in our study and others'. The uncinate fasciculus, which connects orbitofrontal and temporal areas, was not correlated with any of the cognitive test scores in our study.

There were no significant associations between DTI metrics of the association tracts evaluated and whether patients were classified as ALSci. This may reflect the relatively small number of patients in this study meeting the criteria for cognitive impairment, i.e. two subtests at or below the 5 th percentile. Additionally, subgrouping patients into two groups - with or without ALSci - reduces the range of cognitive performance for regression analysis. The clinical significance of the imaging findings in patients with and without behavioral impairment is uncertain. The higher FA and lower RD values in the cingulum observed in these patients could suggest reduced complexity and less mixing of fibers in this region of the cingulum. The cingulum is composed of fibers of varying length connecting specific regions of the cingulate cortex with temporal and parietal regions [61]. The anterior cingulate is activated in tasks of reward, error detection, and conflict monitoring, whereas posterior regions of the cingulum are activated in memory tasks [62]. The behavioral features that lead to the classification of ALSbi are in line with dysfunctions of the anterior cingulate, raising the possibility that loss of fibers between the anterior cingulate and temporal lobes affects the geometry of remaining fibers in the parahippocampal portion of the cingulate bundle. However, this is quite speculative, and it is important to note that DTI measures diffusion of water molecules in various directions and that inferences about white matter microstructure are indirect.

Although several VBM studies have been conducted in ALS patients, to our knowledge no VBM study has been done to date on PLS patients. The VBM analysis confirmed volume loss in regions of the brain related to motor function in both ALS and PLS patients. Volume loss occurred in the right precentral gyrus in ALS patients. This finding is consistent with a metaanalysis that found right precentral gray matter atrophy in ALS across five studies [63], 
although individual reports have described more widespread gray matter loss [16, 64]. In the PLS patients, volume was reduced in the right motor cortex. We did not see diffuse atrophy of subcortical and cortical regions that was described in a study using surface-based volumetric methods [65]. In addition, we did not find any volumetric differences between patients with and without ALSci or ALSbi. The relationship between cognitive functions and central nervous system gray matter structures has been inconclusive in previous studies of ALS [66]. Only one study has demonstrated a direct association between neuropsychological measures and cortical atrophy in ALS: atrophy was found in the frontal, temporal, limbic, and occipital lobes, and was associated with impaired action knowledge [67].

The extent of cognitive impairment and the imaging changes found in patients in this study were very mild. Unlike a number of population studies on ALS, no patient met the criteria for FTLD. This may reflect a sample bias for higher functioning patients. Most of the ALS and PLS participants in this study were college educated and they volunteered to participate in research, which was carried out away from the site of their primary medical care. Another limitation of this study is the relatively small sample size, particularly the number of patients meeting the criteria for ALSci and ALSbi and who were also able to have imaging studies. However, even subtle deficits in executive functioning, affecting speed of processing information or multitasking for example, can affect tasks of daily life.

\section{Conclusion}

We have shown the feasibility of combining neuropsychological tests with a multimodal imaging approach to better understand the mechanisms of cognitive and behavioral impairment in patients with ALS and PLS. This approach sheds light on the relative contributions of disrupted connectivity through white matter tracts and cortical gray matter damage in these conditions. In this study, only changes in diffusion metrics in white matter tracts were associated with a decline in cognitive measures. The involvement of long association fibers supports the theory that cognitive dysfunction can result from an alteration in network connections, including those extending beyond fronto-temporal regions to connections to occipital and parietal areas. Pathology in the white matter tracts connecting these brain regions is likely to impair temporal encoding of information, leading to reduced efficiency of cognitive networks.

\section{Acknowledgements}

This research was supported by the Intramural Research Program of the National Institute of Neurological Disorders and Stroke and the NIH. Dr. Huey was supported by NINDS/NIH grant 5R00NS060766-03. Dr. Russell and the Maryland ALS Clinic database was supported in part by the Department of Veterans Affairs, NIH RR024888, and NINDS/NIH grant U01AR057967-01.

\section{Disclosure Statement}

The authors have no conflicts of interest to disclose. The protocol is registered on clinicaltrials.gov as NCT00334516. 
Meoded et al.: Imaging Findings Associated with Cognitive Performance in Primary Lateral Sclerosis and Amyotrophic Lateral Sclerosis

\section{References}

1 Abrahams S, Leigh PN, Goldstein LH: Cognitive change in ALS: a prospective study. Neurology 2005;64:12221226.

-2 Lomen-Hoerth C, Murphy J, Langmore S, Kramer JH, Olney RK, Miller B: Are amyotrophic lateral sclerosis patients cognitively normal? Neurology 2003;60:1094-1097.

-3 Murphy J, Henry R, Lomen-Hoerth C: Establishing subtypes of the continuum of frontal lobe impairment in amyotrophic lateral sclerosis. Arch Neurol 2007;64:330-334.

- Neary D, Snowden JS, Gustafson L, Passant U, Stuss D, Black S, Freedman M, Kertesz A, Robert PH, Albert M, Boone K, Miller BL, Cummings J, Benson DF: Frontotemporal lobar degeneration: a consensus on clinical diagnostic criteria. Neurology 1998;51:1546-1554.

5 Rascovsky K, Hodges JR, Knopman D, Mendez MF, Kramer JH, Neuhaus J, van Swieten JC, Seelaar H, Dopper EG, Onyike CU, Hillis AE, Josephs KA, Boeve BF, Kertesz A, Seeley WW, Rankin KP, Johnson JK, Gorno-Tempini ML, Rosen H, Prioleau-Latham CE, Lee A, Kipps CM, Lillo P, Piguet O, Rohrer JD, Rossor MN, Warren JD, Fox NC, Galasko D, Salmon DP, Black SE, Mesulam M, Weintraub S, Dickerson BC, Diehl-Schmid J, Pasquier F, Deramecourt V, Lebert F, Pijnenburg Y, Chow TW, Manes F, Grafman J, Cappa SF, Freedman M, Grossman M, Miller BL: Sensitivity of revised diagnostic criteria for the behavioural variant of frontotemporal dementia. Brain 2011; 134:2456-2477.

6 Strong MJ, Grace GM, Freedman M, Lomen-Hoerth C, Woolley S, Goldstein LH, Murphy J, Shoesmith C, Rosenfeld J, Leigh PN, Bruijn L, Ince P, Figlewicz D: Consensus criteria for the diagnosis of frontotemporal cognitive and behavioural syndromes in amyotrophic lateral sclerosis. Amyotroph Lateral Scler 2009;10:131-146.

7 Pringle CE, Hudson AJ, Munoz DG, Kiernan JA, Brown WF, Ebers GC: Primary lateral sclerosis. Clinical features, neuropathology and diagnostic criteria. Brain 1992;115:495-520.

-8 Floeter MK, Mills R: Progression in primary lateral sclerosis: a prospective analysis. Amyotroph Lateral Scler 2009;10:339-346.

-9 Tartaglia MC, Rowe A, Findlater K, Orange JB, Grace G, Strong MJ: Differentiation between primary lateral sclerosis and amyotrophic lateral sclerosis: examination of symptoms and signs at disease onset and during follow-up. Arch Neurol 2007;64:232-236.

10 Caselli RJ, Smith BE, Osborne D: Primary lateral sclerosis: a neuropsychological study. Neurology 1995;45: 2005-2009.

11 Piquard A, Le Forestier N, Baudoin-Madec V, Delgadillo D, Salachas F, Pradat PF, Derouesne C, Meininger V, Lacomblez L: Neuropsychological changes in patients with primary lateral sclerosis. Amyotroph Lateral Scler 2006; 7:150-160.

12 Grace GM, Orange JB, Rowe A, Findlater K, Freedman M, Strong MJ: Neuropsychological functioning in PLS: a comparison with ALS. Can J Neurol Sci 2011;38:88-97.

13 Huey ED, Goveia EN, Paviol S, Pardini M, Krueger F, Zamboni G, Tierney MC, Wassermann EM, Grafman J: Executive dysfunction in frontotemporal dementia and corticobasal syndrome. Neurology 2009;72:453-459.

14 Pereira JM, Williams GB, Acosta-Cabronero J, Pengas G, Spillantini MG, Xuereb JH, Hodges JR, Nestor PJ: Atrophy patterns in histologic vs clinical groupings of frontotemporal lobar degeneration. Neurology 2009; 72:1653-1660.

15 Rohrer JD, Warren JD, Modat M, Ridgway GR, Douiri A, Rossor MN, Ourselin S, Fox NC: Patterns of cortical thinning in the language variants of frontotemporal lobar degeneration. Neurology 2009;72:1562-1569.

16 Chang JL, Lomen-Hoerth C, Murphy J, Henry RG, Kramer JH, Miller BL, Gorno-Tempini ML: A voxel-based morphometry study of patterns of brain atrophy in ALS and ALS/FTLD. Neurology 2005;65:75-80.

17 Grosskreutz J, Kaufmann J, Fradrich J, Dengler R, Heinze HJ, Peschel T: Widespread sensorimotor and frontal cortical atrophy in amyotrophic lateral sclerosis. BMC Neurol 2006;6:17.

18 Abrahams S, Goldstein LH, Suckling J, Ng V, Simmons A, Chitnis X, Atkins L, Williams SC, Leigh PN: Frontotemporal white matter changes in amyotrophic lateral sclerosis. J Neurol 2005;252:321-331.

19 Sarro L, Agosta F, Canu E, Riva N, Prelle A, Copetti M, Riccitelli G, Comi G, Filippi M: Cognitive functions and white matter tract damage in amyotrophic lateral sclerosis: a diffusion tensor tractography study. AJNR Am J Neuroradiol 2011;32:1866-1872.

20 Libon DJ, McMillan C, Avants B, Boller A, Morgan B, Burkholder L, Chandrasekaran K, Elman L, McCluskey L, Grossman M: Deficits in concept formation in amyotrophic lateral sclerosis. Neuropsychology 2012;26:422429.

-21 Brooks BR, Miller RG, Swash M, Munsat TL: El Escorial revisited: revised criteria for the diagnosis of amyotrophic lateral sclerosis. Amyotroph Lateral Scler Other Motor Neuron Disord 2000;1:293-299.

-22 Cedarbaum JM, Stambler N, Malta E, Fuller C, Hilt D, Thurmond B, Nakanishi A: The ALSFRS-R: a revised ALS functional rating scale that incorporates assessments of respiratory function. BDNF ALS Study Group (Phase III). J Neurol Sci 1999;169:13-21.

23 Iwata NK, Kwan JY, Danielian LE, Butman JA, Tovar-Moll F, Bayat E, Floeter MK: White matter alterations differ in primary lateral sclerosis and amyotrophic lateral sclerosis. Brain 2011;134:2642-2655.

24 Jurica SJ, Leitten CL, Mattis S: Dementia Rating Scale-2: Professional Manual. Odessa, Psychological Assessment Resources, 2001.

25 Delis DC, Kramer JH, Kaplan E, Holdnack J: Reliability and validity of the Delis-Kaplan Executive Function System: an update. J Int Neuropsychol Soc 2004;10:301-303. 
Beck AT, Steer RA, Ball R, Ranieri W: Comparison of Beck Depression Inventories -IA and -II in psychiatric outpatients. J Pers Assess 1996;67:588-597.

27 Stout JC, Ready RE, Grace J, Malloy PF, Paulsen JS: Factor analysis of the Frontal Systems Behavior Scale (FrSBe). Assessment 2003;10:79-85.

-28 Cummings JL, Mega M, Gray K, Rosenberg-Thompson S, Carusi DA, Gornbein J: The Neuropsychiatric Inventory: comprehensive assessment of psychopathology in dementia. Neurology 1994;44:2308-2314.

29 Jiang H, van Zijl PC, Kim J, Pearlson GD, Mori S: DtiStudio: resource program for diffusion tensor computation and fiber bundle tracking. Comput Methods Programs Biomed 2006;81:106-116.

-30 Andersson JL, Skare S: A model-based method for retrospective correction of geometric distortions in diffusion-weighted EPI. Neuroimage 2002;16:177-199.

-31 Zhuang J, Hrabe J, Kangarlu A, Xu D, Bansal R, Branch CA, Peterson BS: Correction of eddy-current distortions in diffusion tensor images using the known directions and strengths of diffusion gradients. J Magn Reson Imaging 2006;24:1188-1193.

32 Basser PJ, Mattiello J, LeBihan D: MR diffusion tensor spectroscopy and imaging. Biophys J 1994; 66:259-267.

33 Pierpaoli C, Basser PJ: Toward a quantitative assessment of diffusion anisotropy. Magn Reson Med 1996;36: 893-906.

34 Mori S, Oishi K, Jiang H, Jiang L, Li X, Akhter K, Hua K, Faria AV, Mahmood A, Woods R, Toga AW, Pike GB, Neto PR, Evans A, Zhang J, Huang H, Miller MI, van Zijl P, Mazziotta J: Stereotaxic white matter atlas based on diffusion tensor imaging in an ICBM template. Neuroimage 2008;40:570-582.

-35 Oishi K, Faria A, Jiang H, Li X, Akhter K, Zhang J, Hsu JT, Miller MI, van Zijl PC, Albert M, Lyketsos CG, Woods R, Toga AW, Pike GB, Rosa-Neto P, Evans A, Mazziotta J, Mori S: Atlas-based whole brain white matter analysis using large deformation diffeomorphic metric mapping: application to normal elderly and Alzheimer's disease participants. Neuroimage 2009;46:486-499.

36 Ashburner J: A fast diffeomorphic image registration algorithm. Neuroimage 2007;38:95-113.

-37 Ridgway GR, Henley SM, Rohrer JD, Scahill RI, Warren JD, Fox NC: Ten simple rules for reporting voxel-based morphometry studies. Neuroimage 2008;40:1429-1435.

-38 Bennett CM, Wolford GL, Miller MB: The principled control of false positives in neuroimaging. Soc Cogn Affect Neurosci 2009;4:417-422.

-39 Maldjian JA, Laurienti PJ, Kraft RA, Burdette JH: An automated method for neuroanatomic and cytoarchitectonic atlas-based interrogation of fMRI data sets. Neuroimage 2003;19:1233-1239.

40 Singer MA, Statland JM, Wolfe GI, Barohn RJ: Primary lateral sclerosis. Muscle Nerve 2007;35:291-302.

41 Huey ED, Koppel J, Armstrong N, Grafman J, Floeter MK: A pilot study of the prevalence of psychiatric disorders in PLS and ALS. Amyotroph Lateral Scler 2010;11:293-297.

42 Lomen-Hoerth C, Anderson T, Miller B: The overlap of amyotrophic lateral sclerosis and frontotemporal dementia. Neurology 2002;59:1077-1079.

43 Murphy MJ, Grace GM, Tartaglia MC, Orange JB, Chen X, Rowe A, Findlater K, Kozak RI, Freedman M, Strong MJ, Lee TY: Cerebral haemodynamic changes accompanying cognitive impairment in primary lateral sclerosis. Amyotroph Lateral Scler 2008;9:359-368.

44 Agosta F, Gorno-Tempini ML, Pagani E, Sala S, Caputo D, Perini M, Bartolomei I, Fruguglietti ME, Filippi M: Longitudinal assessment of grey matter contraction in amyotrophic lateral sclerosis: a tensor based morphometry study. Amyotroph Lateral Scler 2009;10:168-174.

45 Sato K, Aoki S, Iwata NK, Masutani Y, Watadani T, Nakata Y, Yoshida M, Terao Y, Abe O, Ohtomo K, Tsuji S: Diffusion tensor tract-specific analysis of the uncinate fasciculus in patients with amyotrophic lateral sclerosis. Neuroradiology 2010;52:729-733.

-46 Zhang Y, Schuff N, Jahng GH, Bayne W, Mori S, Schad L, Mueller S, Du AT, Kramer JH, Yaffe K, Chui H, Jagust WJ, Miller BL, Weiner MW, Zhuang L, Wen W, Zhu W, Trollor J, Kochan N, Crawford J, Reppermund S, Brodaty H, Sachdev P: Diffusion tensor imaging of cingulum fibers in mild cognitive impairment and Alzheimer disease. White matter integrity in mild cognitive impairment: a tract-based spatial statistics study. Neurology 2007;68:13-19.

-47 Song SK, Sun SW, Ramsbottom MJ, Chang C, Russell J, Cross AH: Dysmyelination revealed through MRI as increased radial (but unchanged axial) diffusion of water. Neuroimage 2002;17:1429-1436.

48 Catani M, Howard RJ, Pajevic S, Jones DK: Virtual in vivo interactive dissection of white matter fasciculi in the human brain. Neuroimage 2002;17:77-94.

49 de Zubicaray GI, Rose SE, McMahon KL: The structure and connectivity of semantic memory in the healthy older adult brain. Neuroimage 2011;54:1488-1494.

-50 Kvickstrom P, Eriksson B, van Westen D, Latt J, Elfgren C, Nilsson C: Selective frontal neurodegeneration of the inferior fronto-occipital fasciculus in progressive supranuclear palsy (PSP) demonstrated by diffusion tensor tractography. BMC Neurol 2011;11:13.

51 Latzman RD, Markon KE: The factor structure and age-related factorial invariance of the Delis-Kaplan Executive Function System (D-KEFS). Assessment 2010;17:172-184.

52 Pa J, Possin KL, Wilson SM, Quitania LC, Kramer JH, Boxer AL, Weiner MW, Johnson JK: Gray matter correlates of set-shifting among neurodegenerative disease, mild cognitive impairment, and healthy older adults. J Int Neuropsychol Soc 2010;16:640-650.

-53 Kantarci K, Senjem ML, Avula R, Zhang B, Samikoglu AR, Weigand SD, Przybelski SA, Edmonson HA, Vemuri P, Knopman DS, Boeve BF, Ivnik RJ, Smith GE, Petersen RC, Jack CR Jr: Diffusion tensor imaging and cognitive function in older adults with no dementia. Neurology 2011;77:26-34. 
Meoded et al.: Imaging Findings Associated with Cognitive Performance in Primary Lateral Sclerosis and Amyotrophic Lateral Sclerosis

54 van den Heuvel MP, Hulshoff Pol HE: Exploring the brain network: a review on resting-state fMRI functional connectivity. Eur Neuropsychopharmacol 2010;20:519-534.

55 Metzler-Baddeley C, Jones DK, Steventon J, Westacott L, Aggleton JP, O’Sullivan MJ: Cingulum microstructure predicts cognitive control in older age and mild cognitive impairment. J Neurosci 2012;32:17612-17619.

56 Stuss DT, Levine B: Adult clinical neuropsychology: lessons from studies of the frontal lobes. Annu Rev Psychol 2002;53:401-433.

57 Rafalowska J, Dziewulska D: White matter injury in amyotrophic lateral sclerosis (ALS). Folia Neuropathol 1996;34:87-91.

58 Abrahams S, Goldstein LH, Simmons A, Brammer M, Williams SC, Giampietro V, Leigh PN: Word retrieval in amyotrophic lateral sclerosis: a functional magnetic resonance imaging study. Brain 2004;127:1507-1517.

59 Thiebaut de Schotten M, Dell'Acqua F, Valabregue R, Catani M: Monkey to human comparative anatomy of the frontal lobe association tracts. Cortex 2012;48:82-96.

60 Fine EM, Delis DC, Dean D, Beckman V, Miller BL, Rosen HJ, Kramer JH: Left frontal lobe contributions to concept formation: a quantitative MRI study of performance on the Delis-Kaplan Executive Function System Sorting Test. J Clin Exp Neuropsychol 2009;31:624-631.

61 Beckmann M, Johansen-Berg H, Rushworth MF: Connectivity-based parcellation of human cingulate cortex and its relation to functional specialization. J Neurosci 2009;29:1175-1190.

62 Rushworth MF, Behrens TE: Choice, uncertainty and value in prefrontal and cingulate cortex. Nat Neurosci 2008;11:389-397.

63 Chen Z, Ma L: Grey matter volume changes over the whole brain in amyotrophic lateral sclerosis: a voxel-wise meta-analysis of voxel based morphometry studies. Amyotroph Lateral Scler 2010;11:549-554.

64 Cosottini M, Pesaresi I, Piazza S, Diciotti S, Cecchi P, Fabbri S, Carlesi C, Mascalchi M, Siciliano G: Structural and functional evaluation of cortical motor areas in amyotrophic lateral sclerosis. Exp Neurol 2012;234:169-180.

65 Tartaglia MC, Laluz V, Rowe A, Findlater K, Lee DH, Kennedy K, Kramer JH, Strong MJ: Brain atrophy in primary lateral sclerosis. Neurology 2009;72:1236-1241.

-66 Tsermentseli S, Leigh PN, Goldstein LH: The anatomy of cognitive impairment in amyotrophic lateral sclerosis: more than frontal lobe dysfunction. Cortex 2012;48:166-182.

67 Grossman M, Anderson C, Khan A, Avants B, Elman L, McCluskey L: Impaired action knowledge in amyotrophic lateral sclerosis. Neurology 2008;71:1396-1401. 